Physics

Electricity \& Magnetism fields

\title{
efficient solving techniques of matrix equations for finite element analysis of eddy currents
}

\author{
Takayoshi Nakata \\ N. Takahashi \\ Okayama University \\ Okayama University
}

K. Fujiwara

Okayama University

This paper is posted at eScholarship@OUDIR : Okayama University Digital Information Repository.

http://escholarship.lib.okayama-u.ac.jp/electricity_and_magnetism/7 
EFFICIENT SOLVING TECHNIQUES OF MATRIX EQUATIONS FOR FINITE ELEMENT ANALYSIS OF EDDY CURRENTS

T. Nakata, N. Takahashi and K. Fujiwara

\section{ABSTRACT}

Efficient techniques for solving matrix equations for 2-D finite element analysis of eddy currents in electrical machinery connected to constant voltage sources are conceived by treating grad $\phi$, i.e. $\partial \phi / \partial z$, as unknown variables. The computer storage and the computing time can be considerably reduced by modifying the coefficient matrix to a symmetrical and banded one with edges.

\section{INTRODUCTION}

In 2-D eddy current arialysis using the magnetic vector potential, grad $\phi(\partial \phi / \partial z)$ should be considered in some cases[1]. As the bandwidth of a coefficient matrix in such an analysis becomes large due to $\partial \phi / \partial z$, the computer storage and the computing time are considerably increased[2]. In the finite element analysis of magnetic fields in electrical machinery connected to constant voltage sources, in which the exciting currents are treated as unknown variables, the coefficient matrix becomes unsymmetrical[3]. Though we sometimes encounter such problems, they are not investigated in detail[4].

In this paper, the following new techniques which have been developed to overcome those problems are introduced. The first technique is the reduction of the bandwidth by treating not only the vector potentials but also $\partial \phi / \partial z$ as unknown variables, and the second one is the modification of the unsymmetrical matrix to a symmetrical one.

\section{NEW TECHNIQUES}

\subsection{Reduction of bandwidth}

The new technique is explained using a model with nv windings and ng conductors as shown in Fig.1. Most of the electrical machinery may be represented substantially by this model. Each turn in one winding is connected in series. It is assumed that eddy currents do not flow in the windings. $\mathrm{Vq}$ and Ioq are the terminal voltage and the magnetizing current of the q-th winding respectively.

\section{(1) Fundamental equations}

2-D magnetic fields with eddy currents are governed by the following partial differential equation [5]:

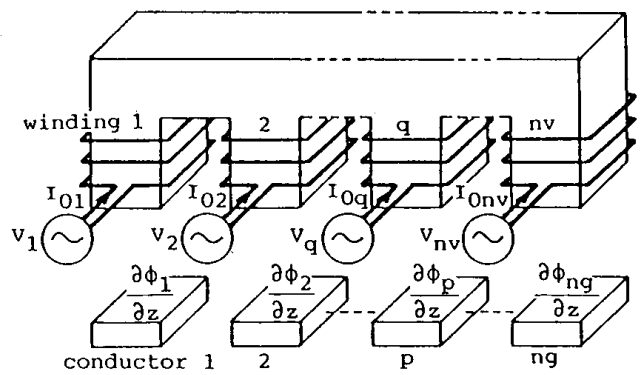

Fig.1 Model with windings and conductors.

The authors are with the Department of Electrical Engineering, Okayama University, Okayama 700, Japan.

$$
\frac{\partial}{\partial x}\left(v \frac{\partial \mathrm{A}}{\partial x}\right)+\frac{\partial}{\partial y}\left(v \frac{\partial \mathrm{A}}{\partial y}\right)=-J_{0}-J_{e}
$$

where $A$ is the magnetic vector potential, and $v$ is the reluctivity. Jo and Je are the magnetizing current density and the eddy current density respectively. Je can be denoted by the vector potential A, the electric scalar potential $\phi$ and the conductivity $\sigma$ as follows [1]:

$$
J_{\mathrm{e}}=-\sigma\left(\frac{\partial \mathrm{A}}{\partial \mathrm{t}}+\frac{\partial \phi}{\partial z}\right)
$$

The following equations can be obtained by Galerkin's method from Eqs. (1) and (2)[3].

$$
\begin{aligned}
& \mathrm{G}_{\mathrm{i}}=\iint_{\Omega} \nu\left(\frac{\partial \mathrm{N}_{\mathrm{i}}}{\partial \mathrm{x}} \frac{\partial \mathrm{A}}{\partial \mathrm{x}}+\frac{\partial \mathrm{N}_{\mathrm{i}}}{\partial \mathrm{y}} \frac{\partial \mathrm{A}}{\partial \mathrm{y}}\right) \mathrm{dxdy} . \\
& -\sum_{q=1}^{n v} \frac{n q}{S_{i v q}} \iint_{\Omega_{q}} N_{i} I \alpha q d x d y \\
& +\sum_{p=1}^{n g} \iint_{\Omega_{p}} N_{i} \sigma \frac{\partial A}{\partial t} d x d y \\
& +\sum_{p=1}^{n g} \iint_{\Omega_{p}} N_{i} \sigma \frac{\partial \phi_{p}}{\partial z} d x d y=0 \\
& (\mathrm{i}=1,2, \cdots, \mathrm{nu}) \\
& (\mathrm{p}=1,2, \cdots, \mathrm{ng}) \\
& (\mathrm{q}=1,2, \cdots, \mathrm{nv})
\end{aligned}
$$

where $\mathrm{Ni}$ is the interpolation function[5], Ioq, Stvq and nq are the magnetizing current, the cross-sectional area and the number of turns of the q-th winding respectively. $\partial \phi_{\mathrm{p}} / \partial z$ is the $\partial \phi / \partial z$ of the $p$-th conductor. $\Omega$ denotes the analyzed region. $\Omega_{\mathrm{p}}$ and $\Omega_{\mathrm{q}}$ are the cross sections of the $p^{-t h}$ conductor and the $q$-th winding respectively. nu is the number of unknown nodes.

\section{(2) Improved method for calculating grad $\phi$}

In the eddy current analysis, an electric field, namely the $\partial \phi / \partial z$ term plays an important role[1]. In 2-D analysis, $\partial \phi / \partial z$ is constant in a conductor, and that of the p-th conductor is given by[1]

$$
\frac{\partial \phi_{p}}{\partial z}=-\frac{1}{S_{t p}} \iint_{\Omega_{p}} \frac{\partial A}{\partial t} d x d y
$$

where Stp is the cross-sectional area of the $\mathrm{p}^{\text {-th }}$ conductor. When there are ng independent conductors in the analyzed region, ng kinds of $\partial \phi / \partial z$ should be considered as shown in Fig. 1.

In the conventional method, the bandwidth is increased when $\partial \phi / \partial z$ is considered[2]. The mechanism of the increase of the bandwidth due to $\partial \phi / \partial z$ is explained using a simple model shown in Fig.2. As the area $\Delta^{(e)}$ of each element $e$ in the conductor is the same, $\partial \phi_{1} / \partial z$ of the conductor in Fig. 2 can be obtained from $\mathrm{Eq}$. (4) as follows :

$$
\begin{aligned}
\frac{\partial \phi_{1}}{\partial z} & =-\frac{1}{S_{t 1}} \frac{\Delta^{(0)}}{3 \Delta t} \times\left(A_{1}+3 A_{2}+3 A_{3}\right. \\
& \left.+2 A_{4}+6 A_{5}+2 A_{6}+3 A_{7}+3 A_{8}+A_{10}\right)
\end{aligned}
$$

0018-9464/88/0100-0170\$01.00@1988 IEEE 


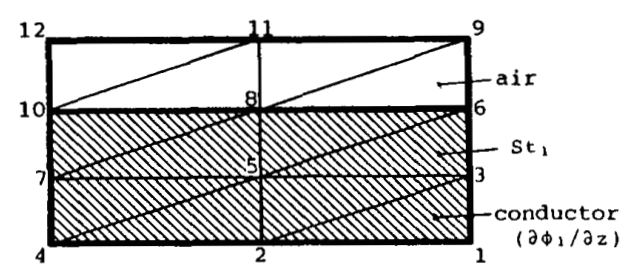

Fig. 2 Model of subdivision.

where $\Delta t$ is the time interval of the time difference method[5]. Stl is the cross-sectional area of the conductor in Fig. 2 .

Equations (3) and (5) suggest that the vector potentials of the nodes on one conductor, such as nodes 1 to 8 and 10 in $\mathrm{Fig.2}$, are related each other. Therefore, the half-bandwidth $\mathrm{Bw}$ of the coefficient matrix is increased as shown in the following equation:

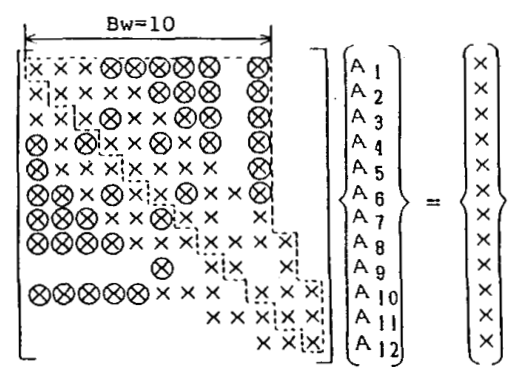

where $x$ in the coefficient matrix denotes the nonzero element and denotes the increased nonzero element due to $\partial \phi / \partial z$. The coefficient matrix is symmetric and forms a banded structure. The half-bandwidth $\mathrm{BW}$ is equal to 10. The fact that $B w$ is equal to 10 means that the matrix in Eq. (6) is nearly the full matrix. Therefore, when the number of unknown nodes is very large, the computer storage and the computing time are considerably increased and the calculation is not easy. If $\partial \phi / \partial z$ is treated as an unknown independent variable, such a disadvantage can be overcome. Because the coefficient matrix becomes the banded one with an edge as shown in the following equation:

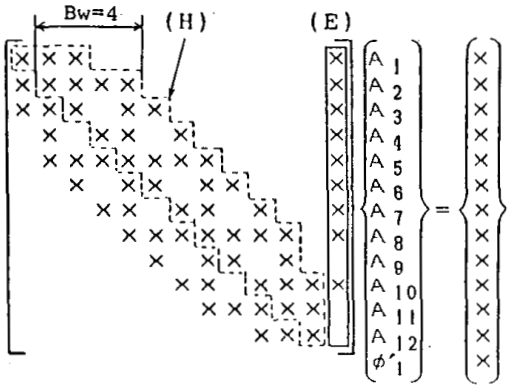

where $\phi$ denotes $\partial \phi / \partial \mathrm{z}$. In $\mathrm{Eq} .(7)$, in $\mathrm{Eq} .(6)$ due to $\partial \phi / \partial z$ are moved to the locations enclosed by a solid line. The number of the columns of the submatrix (E) enclosed by the solid line is equal to that of $\partial \phi / \partial z$, namely the number of conductors. In this case, the number of unknown variables is larger than that of Eq.(6). Therefore, the following equation which is obtained from Eq. (4) should be added to Eq. (6).

$$
\frac{\partial}{\partial z} \frac{\phi_{P}}{z}+\frac{1}{S_{t P}} \iint_{\Omega_{p}} \frac{\partial A}{\partial t} d x d y=0
$$

The final equation for treating $\partial \phi / \partial z$ as unknown variables is giver by

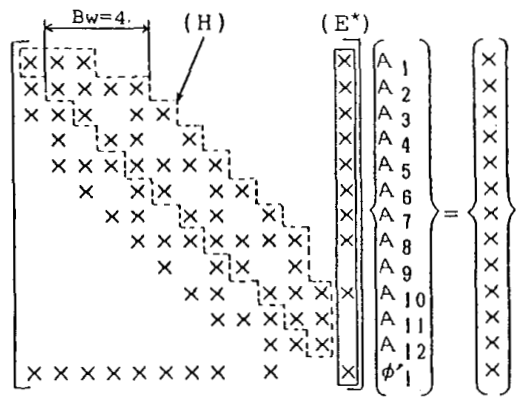

If the coefficient matrix of Eq.(9) is symmetrized as shown in Section 2.2, the calculation is possible by memorizing only the upper part $\left(E^{*}\right)$ of the edges and the banded matrix (H). As only (H) and $\left(\mathrm{E}^{*}\right)$ in $\mathrm{Eq} \cdot(9)$ are memorized and solved independently, the computer storage and the computing time can be reduced[6]. For example, the memory storage is decreased from 62 (locations enclosed by the broken line in Eq. (6)) to 54 (locations enclosed by the broken line $(\mathrm{H})$ and the solid line (E*) in Eq.(9)). The reduction of the computer storage is not so remarkable in this example. when the number of elements, however, is increased and there are many kinds of $\partial \phi / \partial z$, the decrease of computer storage is remarkable. For example, in the case of Fig.4(a) denoted in Section 3, the computer storage is considerably reduced to about $1 / 12$ of that of the conventional method.

In solving Eq. (9), a solver for banded matrix is used for the matrix (H), and that for full matrix is used for the edge matrix ( $\left.E^{*}\right)$ independently.

If the winding has parallel circuits, the number ng of $\partial \phi / \partial z$ are very much increased, because $\partial \phi / \partial z$ should be defined in each turn[7]. As the reduction of the computer storage and the computing time is remarkable when the number of the columns of the edge matrix (E*) in Eq. (9) is large, the technique proposed here is especially effective for such a problem.

(3) Improved method for analyzing electrical machinery connected to constant voltage sources

Magnetic fields in electrical machinery should be analyzed under the specified terminal voltages, because they are usually excited by constant voltage power sources as shown in Fig.1. In such a problem, the terminal voltages $v_{1}, v_{2}, \ldots, v_{n v}$ are given and the magnetizing currents $\mathrm{I}_{01}, \mathrm{I}_{\mathrm{O} 2}, \ldots . . \mathrm{I}_{\mathrm{Onv}}$ are unknown. The analysis of such a problem is possible by treating the magnetizing currents as unknown variables[3].

Figure 3 shows the q-th circuit of Fig.1. The finite element region (2-D) which is enclosed by a broken line in Fig. 3 corresponds to the winding shown in Fig.1 except the coil end. Roq and Loq are the resistance and the leakage inductance of the coil end respectively. Rcq is the resistance of the q-th winding in the finite element region. The following equation can be obtained from Kirchhoff's second law:

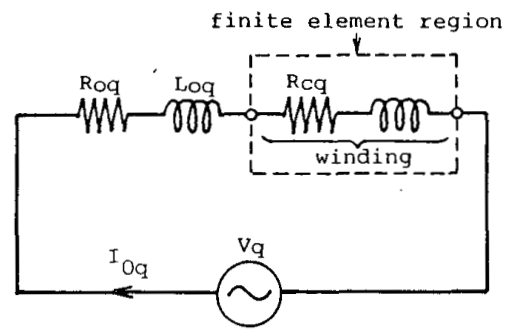

Fig. 3 Equivalent circuit for the q-th circuit. 


$$
\frac{d \Phi_{q}}{d t}+\left(R_{c q}+R_{o q}\right) I_{o q}+L_{o q} \frac{d I_{o q}}{d t}-V_{q}=0
$$

where $\Phi_{\mathrm{q}}$ is the interlinkage flux of the winding. By representing the interlinkage flux $\Phi_{\mathrm{q}}$ using the vector potential A, Eq.(10) can be rewritten as follows:

$$
\begin{aligned}
& \frac{n_{q}}{S_{1 \vee q}} \iint_{\Omega_{q}} \frac{\partial A}{\partial t} d x d y+\left(R_{c q}+R_{o q}\right) I_{o q} \\
& +L_{o q} \frac{d I \text { Iq }}{d t}-V_{q}=0
\end{aligned}
$$

As there are nv independent windings, the number of equations similar to Eq.(11) is nv.

As the number of Eq. (3) and that of Eq.(11) are nu and nv respectively, both the vector potentials and the magnetizing currents can be calculated by solving those equations simultaneously. The final equations for analyzing eddy currents in electrical machinery connected to constant voltage sources can be obtained by combining Eq.(3) with Eqs. (8) and (11). In the nonlinear analysis using the Newton-Raphson iteration technique, the increments $\delta A_{j}, \delta \phi_{k}^{\prime}$ and $\delta I_{o} \ell$ at the instant $t$ are obtained from the following equation:

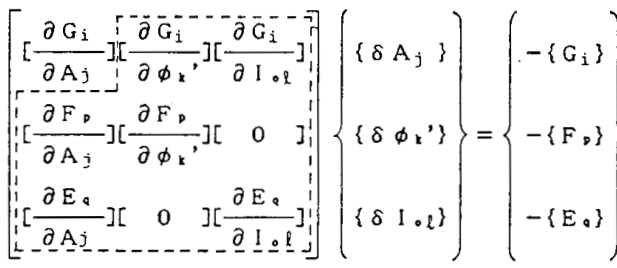

$$
\begin{aligned}
& \left(\begin{array}{l}
j=1,2, \ldots, n u \\
k=1,2, \ldots, n g \\
\ell=1,2, \ldots, n v
\end{array}\right)
\end{aligned}
$$

For the first-order finite element method, $\mathrm{Gi}$ is given by[5]

$$
\begin{aligned}
& \mathrm{G}_{i}=\sum_{\mathrm{e}=1}^{\mathrm{Ne}}\left\{v^{(0)} \sum_{k=1}^{3} \mathrm{~S}_{i k_{0}} \mathrm{~A}_{\mathrm{k}}^{\mathrm{t}}\right. \\
& \left.+\sigma \sum_{k=1}^{3} \frac{\Delta^{(0)}}{12}\left(1+\delta_{1 k_{0}}\right) \frac{A_{k_{0}}^{t}-A_{k_{\ell}}^{t-\Delta t}}{\Delta t}\right) \\
& -\sum_{q=1}^{n v} \frac{n_{q}}{S_{t v q}} \sum_{e=1}^{N C_{q}} \frac{\Delta^{(0)}}{3} \delta_{i}^{(0)} I_{o q} \\
& +\sum_{p=1}^{n g} \sigma \sum_{e=1}^{N_{p}} \frac{\Delta^{(0)}}{3} \phi_{p}^{\prime}
\end{aligned}
$$

where $\mathrm{Ne}$ is the number of elements in the whole region. Ncq and $\mathrm{Np}$ are the number of elements in the cross section of the $q$-th winding and that in the p-th conductor respectively. $A_{k e}$ is the vector potential at a node ke. The superscript $t$ denotes the instant of the calculation. $\delta_{i k e}$ is the kronecker delta. $\delta_{i}$ (e) is unity when the node $i$ is in the element $e$ and zero when the node $i$ is outside the element e. $S_{i k e}$ is defined by[5]

$$
S_{1 k \theta}=\frac{1}{4 \Delta^{(\theta)}}\left(c_{i}^{(\theta)} c_{k \theta}+d_{i}^{(\theta)} d_{k \theta}\right)
$$

$c_{k e}$ and $d_{k e}$ are denoted by

$$
\left.\begin{array}{l}
c_{k e}=y_{i e}-y_{j e} \\
d_{k e}=x_{j e}-x_{i e}
\end{array}\right\}
$$

with the other coefficients obtained by a cyclic permutation by subscripts in the order $i, j, k$.
Fp and Eq correspond to the left sides of Eqs.(8) and (11) as follows:

$$
\begin{aligned}
F_{p}= & \frac{\partial \phi_{p}}{\partial z}+\frac{1}{S_{t p}} \iint_{\Omega_{p}} \frac{\partial A}{\partial t} d x d y \\
E_{q}= & \frac{n_{q}}{S_{t \vee q}} \iint_{\Omega_{q}} \frac{\partial A}{\partial t} d x d y+\left(R_{c q}+R_{o q}\right) I_{\sigma q} \\
& +L_{o q} \frac{d I \text { I }}{d t}-V_{q}
\end{aligned}
$$

$\partial G_{i} / \partial A_{j}$ etc. in Eq. (12) are derived from Eqs. (13), (16) and (17).

\subsection{Symmetrization of Matrix}

The coefficient matrix in Eq.(12) is not symmetric in the region enclosed by a broken line. That is, $\partial G_{i} / \partial \phi_{k}^{\prime}$ in Eq.(12) is different from $\partial F_{p} / \partial A_{j}$ as follows :

$$
\begin{aligned}
& \frac{\partial \mathrm{G}_{1}}{\partial \phi_{\mathrm{k}}{ }^{\prime}}=\sigma \sum_{\mathrm{e}-1}^{N p} \frac{\Delta^{(0)}}{3} \delta_{i}^{(0)} \\
& \frac{\partial \mathrm{F}_{\mathrm{p}}}{\partial \mathrm{A}_{\mathrm{j}}}=\frac{1}{S_{\mathrm{tp}} \Delta t} \sum_{\mathrm{e}-1}^{N p} \frac{\Delta^{(0)}}{3} \delta_{j}^{(0)}
\end{aligned}
$$

$\partial \mathrm{G}_{\mathrm{i}} / \partial \mathrm{I}_{\mathrm{Ol}}$ is also different from $\partial \mathrm{E}_{\mathrm{q}} / \partial \mathrm{A}_{\mathrm{j}}$ as follows:

$$
\begin{aligned}
& \frac{\partial \mathrm{G}_{1}}{\partial \mathrm{I}_{\mathrm{o}}}=-\frac{\mathrm{n}_{\mathrm{q}}}{\mathrm{S}_{\mathrm{tvq}}} \sum_{\mathrm{e}=1}^{\mathrm{NCq}} \frac{\Delta(0)}{3} \delta_{i}^{(0)} \\
& \frac{\partial \mathrm{E}_{\mathrm{q}}}{\partial \mathrm{A}_{\mathrm{j}}}=-\frac{\mathrm{n}_{\mathrm{q}}}{\mathrm{S}_{\mathrm{tvq}} \Delta \mathrm{t}} \sum_{e=1}^{N C q} \frac{\Delta(e)}{3} \delta_{j}^{(0)}
\end{aligned}
$$

If Eqs. (19) and (21) are multiplied by $s_{t p \Delta t \sigma}$ and $\Delta t$ respectively, the edges of Eq.(12) become symmetric. As $\left[\partial G_{i} / \partial A_{j}\right]$ in $E_{*} \cdot(12)$ is a banded matrix, Eq.(12) can be solved as a banded matrix with symmetrical edges. Therefore, the computer storage and the computing time can be reduced.

\section{AN EXAMPLE}

In order to verify the usefulness of the new technique, it is applied to models shown in Fig.4. Each turn in one winding is connected in series. Six isolated conductors or three isolated ring conductors which are infinitely long in the $\mathrm{z}$-direction are placed inside an exciting winding. The exciting winding is connected to an external power source $V_{1}$. The 2-D construction for Figs.4(a) and (b) are the same as shown in Fig.5. We assune that the model is surrounded by the high permeability material $(\mu=\infty)$ as denoted by the hatched part in Fig.5. Then, the line c0 is the Dirichlet boundary $(A=0)$ and the line $0-a-b-c$ is the Neumann boundary. Though $\partial \phi / \partial z$ can be neglected in Fig.4(b), it should be defined in each conductor in the case of Fig.4(a)[1]. ng and nv in Eq.(12) are 3 and 1 respectively in the case of Fig.4(a), and they are zero and 1 respectively in the case of Fig.4(b). The conductivity o of the conductor is $0.354 \times 10^{8}(\mathrm{~S} / \mathrm{m})$. The resistance ( $R c q+R o q$ ) of the winding is $I(\Omega)$ and the leakage inductance Loq is neglected. The frequency and the effective voltage of the external power source are $50(\mathrm{~Hz})$ and $100(\mathrm{~V})$ respectively. The number of elements and that of unknown nodes are 4608 and 2352 respectively.

The flux distribution in Fig.4(a) is different from that in Fig.4(b) as shown in Fig.6. Table 1 shows the comparison of the half-bandwidth, the computer storage required for the coefficient matrix and the computing time. This Table shows that they can be considerably reduced by introducing the new techniques. Even if there are three kinds of $\partial \phi / \partial z$ as shown in Fig.4(a), the analysis is possible within only a small increase of the memory storage and the computing 


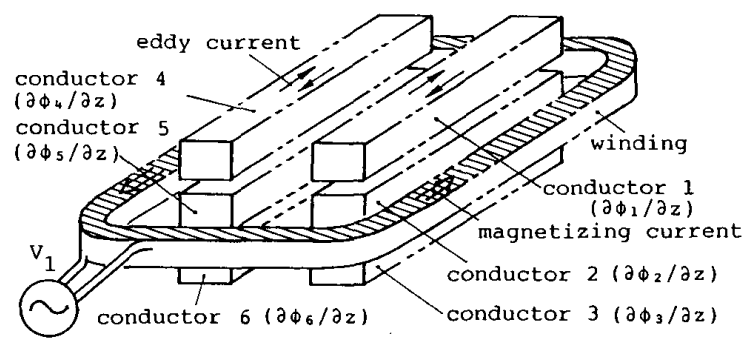

(a) six parallel conductors which are not connected each other

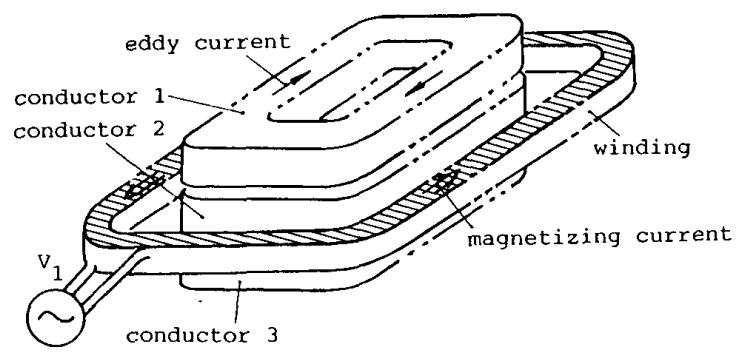

(b) three parallel ring conductors

Fig. 4 Analyzed models.

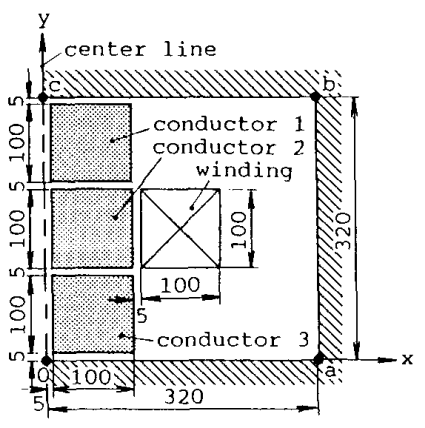

c-O : Dirichlet boundary $(A=0)$

$0-a-b-c:$ Neumann boundary

Fig. 5 Sectional view of analyzed model.

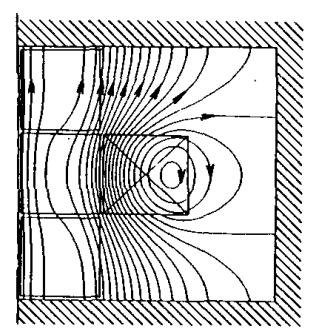

(a) with $\partial \phi / \partial z$

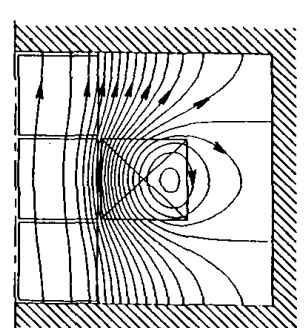

(b) without $\partial \phi / \partial z$
Fig. 6 Effects of $\partial \phi / \partial z$ on flux distributions.
Table 1 Comparison of half-bandwidth, memory storage and CPU time

\begin{tabular}{|c|c|c|c|c|}
\hline Model & $\begin{array}{l}\text { Type of } \\
\text { matrix }\end{array}$ & $\begin{array}{c}\text { Half- } \\
\text { band- } \\
\text { width } \\
\text { Bw }\end{array}$ & $\begin{array}{l}\text { Memory } \\
\text { storage } \\
(k w)\end{array}$ & $\begin{array}{l}\text { CPU } \\
\text { time } \\
\text { (sec.) }\end{array}$ \\
\hline \multirow{2}{*}{$\begin{array}{l}\text { Fig. 4(a) } \\
(w i t h \\
\partial \phi / \partial z)\end{array}$} & $\begin{array}{l}\text { Conventional } \\
(n v=1)\end{array}$ & 1536 & 1355 & 3562 \\
\hline & $\begin{array}{l}\text { With symmetrical } \\
\text { edges }(\mathrm{nv}=1, \mathrm{ng}=3)\end{array}$ & 73 & 111 & 30 \\
\hline $\begin{array}{c}\text { Fig. } 4(b) \\
\text { (without } \\
\partial \Phi / \partial z \text { ) }\end{array}$ & $\begin{array}{l}\text { With symmetrical } \\
\text { edges (nv=1) }\end{array}$ & 73 & 104 & 27 \\
\hline
\end{tabular}

Computer : NEC ACOS-1000 (15MIPS)

time compared with those of the problem having no $\partial \phi / \partial z(F i g .4(b))$.

\section{CONCLUSTONS}

It has been shown that the computer storage and the computing time can be considerably reduced, for example, to about $1 / 10$ and $1 / 100$ of the conventional ones, by treating $\partial \phi / \partial z$ as unknown variables and introducing symmetrization technique. If the number of $\partial \phi / \partial z$ is increased, the reduction of the computer storage and the computing time is more remarkable.

The techniques proposed here are more effective in 3-D analysis. The study in 3-D analysis will be reported later.

\section{REFERENCES}

[1] T.Nakata, N.Takahashi and K.Fujiwara : "Physical Meaning of gradф in Eddy Current Analysis Using Magnetic vector Potentials", Compumag Conference, Graz (1987).

[2] T.Nakata, N.Takahashi and Y.Kawase : "Efficient Calculation Method of Matrix in the Finite Element Analysis", Papers of Technical Meeting of Information Processing, IEE of Japan, IP-80-50 (1980).

[3] T.Nakata and N.Takahashi: "Direct Finite Element Analysis of Flux and Current Distributions under Specified Conditions", IEEE Trans. Magnetics, MAG$18,2,325$ (1982).

[4] J.Weiss and z.Cendes : "Efficient Finite Element Solution of Multipath Eddy Current Problems", ibid. MAG-18, 6, 1710 (1982).

[5] T.Nakata and N.Takahashi: "Finite Element Method in Electrical Engineering",(book), Morikita Publishing Co., Tokyo (1982).

[6] T.Nakata, Y.Ishihara and N.Takahashi : "Some Useful Techniques on Implementing the Finite Element Method for Computation of Electromagnetic Fields in Electrical Machinery", in Interdisciplinary Finite Element Analysis (J.F.Abel, T.Kawai and S.F.Shen, Eds.), 545, Cornell University (1981).

[7] T.Nakata, N.Takahashi and Y.Kawase: "Analysis of Eddy Currents (Circulating Currents) in Cylindrical Parallel Windings", Papers of Technical Meeting of Static Apparatus, IEE of Japan, SA-80-4 (1980). 